\title{
Theoretical Investigations on the Potential-Induced Formation of Pt-Oxide Surfaces
}

\author{
Timo Jacob* \\ Fritz-Haber-Institut der Max-Planck-Gesellschaft, \\ Faradayweg 4-6, D-14195 Berlin, Germany
}

\begin{abstract}
Using the extended $a b$ initio atomistic thermodynamics approach together with density functional theory calculations the interfacial structure and composition of Pt-electrodes in electrochemical environments at elevated electrode potentials was studied. Focusing on the electrode potential region, at which the oxide-formation occurs, the bulk systems and all lowindex surfaces of $\alpha-\mathrm{PtO}_{2}, \beta-\mathrm{PtO}_{2}$, and $\mathrm{PtO}$ bulk-oxides were calculated. On the basis of the bulk-oxide formation energies we first deduced the stability ranges at which the bulk-oxides are the thermodynamically stable phases. In agreement with experimental observations, we find that at experimental temperature and pressure conditions $\alpha-\mathrm{PtO}_{2}$ and $\beta-\mathrm{PtO}_{2}$ bulk-oxides are stable above $1.2 \mathrm{~V}$, while PtO requires $\Delta \phi>1.3 \mathrm{~V}$. Afterwards the corresponding $(p, T, \phi)$ phase diagrams of surface structures were obtained, showing a preference for $\alpha-\mathrm{PtO}_{2}(001), \beta$ $\mathrm{PtO}_{2}(110)$, and $\mathrm{PtO}(100)$ respectively, having bulk-like compositions even on their surfaces. However, in case of thin oxide layers a PtO composition might also be present.
\end{abstract}

Keywords: Density Functional Theory, Pt, Oxides, Electrochemistry, Electrooxidation *corresponding author: jacob@fhi-berlin.mpg.de

\section{Introduction}

Understanding the structure and composition of electrode/electrolyte interfaces (e.g. metal/water) in an electrochemical environment and under realistic conditions remains a crucial aspect in surface electrochemistry. One of the important parameters is certainly the electrode potential, which may significantly influence the electronic and geometric configuration of the interface. Besides the accumulation of excess charge on the electrode surface, which modifies the electronic structure, potential-dependent phenomena can be induced, such as adsorption processes [1,2], surface reconstructions $[3,4,5]$, or even complex-formations $[6,7]$.

With respect to the growth of oxides on metal electrodes by electrooxidation, experimental cyclicvoltammetry (CV) measurements in high-purity electrolytes allow one to distinguish between pronounced potential regions, which can be assigned to an initial interface charging, followed by surface oxidation, and finally the oxide formation at higher positive electrode potentials.

Although different experimental techniques, such as $\mathrm{CV}$, electrochemical quartz-crystal nanobalance (EQCN) or Auger electron spectroscopy (AES) have been employed in order to obtain a better understanding of these structural changes, even for the "standard" system of a Pt electrode in an aqueous solution (e.g. $\mathrm{H}_{2} \mathrm{SO}_{4}$ ) there is still an ongoing debate on the geometry and composition on the atomic scale. While it was commonly accepted that during electrooxidation of a $\mathrm{Pt}$ electrode first $\mathrm{OH}$ is adsorbing on the surface, which at higher positive electrode potentials is used to grow an oxide $[8,9,10]$, recent experiments by Jerkiewicz et al. [11] showed no evidence for an adsorbed $\mathrm{OH}$ species. Instead by calculating a molecular weight of the adsorbing species of $15.8 \mathrm{~g} \mathrm{~mol}^{-1}$ from EQCN and CV measurements, they concluded the presence of atomic oxygen only $(0.85 \mathrm{~V}<\Delta \phi<1.1 \mathrm{~V})$. Interestingly Stalnionis et al. [12] found a similar mass increase at potentials within the surface oxidation region, but interpreted those as adsorbing $\mathrm{OHs}$.

Similarly there are also uncertainties regarding the structure of the Pt bulk-oxide, which experimentally was found to form at potentials above $1.0-1.2 \mathrm{~V}$ (depending on the experimental conditions). Here the common view is that oxide-growth first begins with the formation of a thin layer having a PtO composition, on which a $\mathrm{PtO}_{2}$-composed oxide continues growing $[13,14,15]$.

While for surfaces under UHV conditions, experimental techniques have been developed to study their structures on the atomic scale, this is significantly more difficult to achieve under electrochemical conditions. Thus, there is a strong need for theoretical methods to complement experimental attempts aiming at understanding the fundamental phenomena occurring at electrochemical interfaces. An overview of previous and actual theoretical investigations dealing with electrochemical systems can be found in Ref. [16]. In this context, we recently formulated the extended $a b$ initio atomistic thermodynamics method, which allows one to calculate the $(p, T, \phi)$-stability phase diagrams from first principles.

While the region of lower positive potentials, corresponding to the interface charging and the oxygen 
adsorption on the electrode surface, is subject of a separate publication [17], the present work aims at understanding the structure and composition of the Ptelectrode at higher electrode potentials at which the formation of surface/bulk-oxides occurs.

In the following we will first briefly describe the extended $a b$ initio atomistic thermodynamics approach, which then will be adapted to the system of a Pt bulkoxide in contact with an aqueous solution. Using the energetics of the corresponding bulk-calculations these $(p, T, \phi)$-conditions are determined under which the bulkoxides are thermodynamically stable. Finally the $(p, T, \phi)$ surface phase diagrams of the three stable bulk-oxides are discussed, giving insights into the oxide surface structures under various environmental conditions.

\section{Methods \\ 2.1. DFT-Calculations}

For the calculations on the different Pt-oxide surfaces SeqQuest $[18,19]$, a periodic DFT program with localized basis sets represented by a linear combination of Gaussian functions, was used together with the PBE [20] Generalized Gradient Approximation (GGA) exchange-correlation functional. A standard (non-local) norm-conserving pseudopotential [21] was applied to replace the 62 core electrons of each $\mathrm{Pt}$, leaving the $5 \mathrm{~d}$ and 6 s-electrons in the valence space and invoking a nonlinear core correction [22]. The basis sets were optimized "double zeta plus polarization" contracted Gaussian functions.

Prior to constructing the surface structures, the corresponding bulk systems (see Fig. 1) were calculated. In each case the bulk unitcell was optimized and the atom positions relaxed, leading to the bulk crystal structures and formation energies summarized in Table 1. While experimentally $\mathrm{Pt}_{3} \mathrm{O}_{4}$ was found to be a stable phase at higher pressures the optimized structures for $\alpha-\mathrm{PtO}_{2}, \beta$ $\mathrm{PtO}_{2}$, and $\mathrm{PtO}$ are in agreement with experiments. In addition, the formation energy per $\mathrm{O}$-atom of $-0.72 \mathrm{eV}$ for the most stable bulk Pt oxides $\left(\alpha-\mathrm{PtO}_{2}\right.$ and $\left.\beta-\mathrm{PtO}_{2}\right)$, calculated by

$$
E=\frac{1}{y}\left(E_{\mathrm{Pt}_{\mathrm{x}} \mathrm{O}_{\mathrm{y}}}^{\text {bulk }}-x \cdot E_{\mathrm{Pt}}^{\text {bulk }}-\frac{y}{2} \cdot E_{\mathrm{O}_{2}}^{\mathrm{gas}}\right),
$$

corresponds to the experimental value of $-0.69 \mathrm{eV}$ [23] and calculations by Li et al. [24].

On the basis of the calculated crystal structures all low-index surfaces of the three stable Pt bulk-oxides were constructed as symmetric slabs. Therefore, besides the central layer, all atoms have been included in the geometry optimization procedure (to $<0.01 \mathrm{eV} / \AA$ ). Moreover, for each surface all possible surface terminations have been considered. Finally for every system the number of layers as well as the vacuum between periodically repeated slabs was fully converged.

\subsection{Thermodynamic Considerations}

Considering the non-electrochemical system, in which a Pt surface is in contact with an oxygen atmosphere, the stability of the interface is given by the surface Gibbs energy [25]:

$$
\begin{aligned}
\gamma\left(T, p_{\mathrm{Pt}}, p_{\mathrm{O}_{2}}\right)=\frac{1}{A}[G & -N_{\mathrm{Pt}} \mu_{\mathrm{Pt}}\left(T, p_{\mathrm{Pt}}\right) \\
& \left.-N_{\mathrm{O}_{2}} \mu_{\mathrm{O}_{2}}\left(T, p_{\mathrm{O}_{2}}\right)\right],
\end{aligned}
$$

where $A$ is the surface area (in case of the slab geometry both the top and bottom layers have to be considered), $G$ the Gibbs energy of the slab containing $N_{\mathrm{Pt}}$ platinum atoms and $N_{\mathrm{O}}=2 N_{\mathrm{O}_{2}}$ oxygen atoms with the corresponding chemical potentials $\mu_{\mathrm{Pt}}$ and $\mu_{\mathrm{O}_{2}}$ being functions of temperature and partial pressures. If we now assume the presence of macroscopic quantities of bulk $\mathrm{Pt}_{x} \mathrm{O}_{y}$, which should be in equilibrium with the $\mathrm{O}_{2}$ environment, the condition

$$
x \cdot \mu_{\mathrm{Pt}}+\frac{y}{2} \cdot \mu_{\mathrm{O}_{2}}=g_{\mathrm{Pt}_{\mathrm{x}} \mathrm{O}_{\mathrm{y}}}^{\text {bulk }}
$$

allows us to introduce the Gibbs energy of the bulk oxide $g_{\mathrm{Pt}_{\mathrm{x}} \mathrm{O}_{\mathrm{y}}}^{\text {bulk }}\left(T, p_{\mathrm{Pt}_{\mathrm{x}} \mathrm{O}_{\mathrm{y}}}\right)$, which due to the solid character of the bulk-oxide will show minor temperature and pressure dependence. With eq. (3) the surface Gibbs energy of the interface becomes

$$
\gamma\left(T, p_{\mathrm{O}_{2}}\right)=\frac{1}{A}\left[G-\frac{N_{\mathrm{Pt}}}{x} g_{\mathrm{Pt}_{\mathrm{x}} \mathrm{O}_{\mathrm{y}}}^{\text {bulk }}+\left(y \frac{N_{\mathrm{Pt}}}{x}-N_{\mathrm{O}}\right) \mu_{\mathrm{O}}\right] \text {. }
$$

Since the bulk-oxide is assumed to be in contact with an $\mathrm{O}_{2}$ atmosphere the chemical potential of $\mathrm{O}$, which enters into eq. (4) is determined by the condition of thermodynamic equilibrium with the surrounding $\mathrm{O}_{2}$ gasphase reservoir: $\mu_{\mathrm{O}}=(1 / 2) \mu_{\mathrm{O}_{2}}$. Consequently, its temperature and pressure dependence is given by

$$
\mu_{\mathrm{O}}\left(T, p_{\mathrm{O}_{2}}\right)=\frac{1}{2}\left[E_{\mathrm{O}_{2}}^{\text {tot }}+\bar{\mu}_{\mathrm{O}_{2}}\left(T, p^{0}\right)+k_{\mathrm{B}} T \ln \left(\frac{p_{\mathrm{O}_{2}}}{p^{0}}\right)\right] \text {. }
$$

In order to move from gas-phase to the electrochemical system, we recently extended the $a b$ initio atomistic thermodynamics approach [17], which in principle allows calculating the stability of electrode/electrolyte interfaces under electrochemical conditions.

If we assume that every oxygen desorbing from the bulk-oxide electrode $\left(\mathrm{O}_{\text {oxide }}\right)$ forms water by reacting with two protons from the electrolyte being at the electrostatic potential $\phi_{\mathrm{s}}$ and two electrons from the electrode being at the potential $\phi_{\mathrm{e}}$

$$
\mathrm{O}_{\text {oxide }}+2 \mathrm{H}^{+}+2 \mathrm{e}^{-} \leftrightarrow \mathrm{H}_{2} \mathrm{O}(1)
$$

then analogous to eq. (4) the stability of different electrode/electrolyte interface structures can be evaluated by comparing the interfacial (here surface) free energy:

$$
\begin{aligned}
\gamma\left(T, a_{\mathrm{H}_{2} \mathrm{O}}, \Delta \phi\right) & =\frac{1}{A}\left[G\left(T, a_{\mathrm{H}_{2} \mathrm{O}}, N_{e}\right)-\frac{N_{\mathrm{Pt}}}{x} g_{\mathrm{Pt}_{\mathrm{x}} \mathrm{O}_{\mathrm{y}}}^{\text {bulk }}\right. \\
& \left.+\left(y \frac{N_{\mathrm{Pt}}}{x}-N_{\mathrm{O}}\right) \cdot\left(\mu_{\mathrm{H}_{2} \mathrm{O}}+2 e \Delta \phi\right)\right] .
\end{aligned}
$$


Compared to eq. (4) the interfacial free energy is now dependent on temperature, activity of the surrounding liquid water, and the difference between the electrostatic potentials of electrode and electrolyte $\Delta \phi=\phi_{\mathrm{e}}-\phi_{\mathrm{s}}$. In addition the oxygen chemical potential has been replaced by $\mu_{\mathrm{O}}=\mu_{\mathrm{H}_{2} \mathrm{O}}+2 e \Delta \phi$, is water the source of any $\mathrm{O}^{2-}$. Although in principle eq. (6) is exact, the Gibbs energy $G$ corresponds to the entire interface, which would require a self-consistent modeling of the full electric double-layer. Depending mainly on temperature, pressure, electrode potential, and ion concentration within the electrolyte, the extension of this interfacial region may vary from a few $\AA$ to more than $100 \mathrm{~nm}$, thus resulting in systems too large for quantum-mechanical calculations. However, if the following assumptions are made (see Ref. [17] for further details):

1. the binding energies of adsorbates on the electrode surface should be independent of the electrode potential $\phi_{\mathrm{e}}$, respectively the number of excess electrons on the electrode surface $N_{\mathrm{e}}$;

2. the electrolyte is fixed and has a constant influence on the electrode surface,

then eq. (6) reduces to:

$$
\begin{aligned}
\gamma\left(T, a_{\mathrm{H}_{2} \mathrm{O}}, \Delta \phi\right) & =\frac{1}{A}\left[G_{\text {slab }}^{\text {surf }}-\frac{N_{\mathrm{Pt}}}{x} g_{\mathrm{Pt}_{\mathrm{x}} \mathrm{O}_{\mathrm{y}}}^{\text {bulk }}\right. \\
& \left.+\left(y \frac{N_{\mathrm{Pt}}}{x}-N_{\mathrm{O}}\right) \cdot\left(\mu_{\mathrm{H}_{2} \mathrm{O}}+2 e \Delta \phi\right)\right] .
\end{aligned}
$$

Since our aim is in comparing interfacial stabilities, here $G_{\text {slab }}^{\text {surf }}$ is the Gibbs energy of the Pt-oxide surface, which due to the two assumptions described above can be evaluated without the presence of the electrolyte now. In addition, because the $\mathrm{Pt}_{\mathrm{x}} \mathrm{O}_{y}$ electrode is present in the solid phase, it is reasonable to assume the $T$ and $p / a$ dependence of $G_{\text {slab }}^{\text {surf }}$ and $g_{\mathrm{Pt}_{\mathrm{x}} \mathrm{O}_{\mathrm{y}}}^{\text {bul }}$ to be rather small, that is why the DFT-energies, which mimic $T=0 \mathrm{~K}$, were used instead.

Although the assumptions mentioned before are quite strong, they represent the actual level of modeling. Consequently almost all theoretical work, that tries to tackle the problem of including the electrode potential without providing a self-consistent description of the entire electric double-layer, make use of these assumptions, but they are barely mentioned.

While above equations were derived for a single electrochemical half cell, the presence of a reversible counter electrode (e.g. reversible hydrogen electrode) would compensate each proton generated during the splitting of water to produce $\mathrm{O}^{2-}$ by an immediate hydrogen evolution reaction. As a consequence the oxygen chemical potential, which entered eq. (4), would then also be related to the chemical potential of the gaseous hydrogen [26]:

$$
\mu_{\mathrm{O}}=\mu_{\mathrm{H}_{2} \mathrm{O}}-1 / 2 \mu_{\mathrm{H}_{2}}+2 e \Delta \phi,
$$

where $\Delta \phi$ now is the potential difference between the electrode of interest and the reversible counter electrode.
Since this would introduce an additional dependency of the interfacial free energy $\gamma$ on the hydrogen partial pressure $p_{\mathrm{H}_{2}}$, in the following we concentrate on the electrochemical half cell. However, the phase diagrams discussed later can easily be transferred to the full system by exchanging the $\mu_{\mathrm{H}_{2} \mathrm{O}}$-labels with $\mu_{\mathrm{H}_{2} \mathrm{O}}-1 / 2 \mu_{\mathrm{H}_{2}}$.

\subsection{Stability Condition}

As our interest lies in the region of higher positive electrode potentials, where the bulk-oxide is the thermodynamically stable phase, we have to analyze the conditions under which the $\mathrm{Pt}_{x} \mathrm{O}_{y}$ bulk-oxide does not decompose. For the non-electrochemical system of a $\mathrm{Pt}$ surface in contact with an $\mathrm{O}_{2}$-atmosphere the oxide is only stable if

$$
g_{\mathrm{Pt}_{\mathrm{x}} \mathrm{O}_{\mathrm{y}}}^{\text {bulk }}<x \cdot g_{\mathrm{Pt}}^{\text {bulk }}+\frac{y}{2} \cdot \mu_{\mathrm{O}_{2}} \text {. }
$$

In the electrochemical system water is the source of all oxygens, which together with two electrons are used to form the oxide. Therefore, the analog of eq. (9) under electrochemical conditions is

$$
g_{\mathrm{Pt}_{\mathrm{x}} \mathrm{O}_{\mathrm{y}}}^{\text {bulk }}<x \cdot g_{\mathrm{Pt}}^{\text {bulk }}+y \cdot\left(\mu_{\mathrm{H}_{2} \mathrm{O}}+2 e \Delta \phi-\Delta G_{\mathrm{O}_{2}}^{\mathrm{H}_{2} \mathrm{O}}\right),
$$

where $\Delta G_{\mathrm{O}_{2}}^{\mathrm{H}_{2} \mathrm{O}}$ is the Gibbs energy required to form $1 / 2 \mathrm{O}_{2}$ out of liquid water (exp.[27]: $2.46 \mathrm{eV}$ ). Equation (10) can be rewritten as

$$
\begin{array}{r}
\Delta \mu_{\mathrm{H}_{2} \mathrm{O}}>\frac{1}{y}\left[g_{\mathrm{Pt}_{\mathrm{x}} \mathrm{O}_{\mathrm{y}}}^{\text {bulk }}-x \cdot g_{\mathrm{Pt}}^{\text {bulk }}-\frac{y}{2} E_{\mathrm{O}_{2}}^{\text {tot }}\right] \\
-2 e \Delta \phi+\Delta G_{\mathrm{O}_{2}}^{\mathrm{H}_{2} \mathrm{O}},
\end{array}
$$

where we used $\Delta \mu_{\mathrm{H}_{2} \mathrm{O}}=\mu_{\mathrm{H}_{2} \mathrm{O}}-\frac{1}{2} E_{\mathrm{O}_{2}}^{\text {tot }}$. This referencing of the water chemical potential by half the total energy of an $\mathrm{O}_{2}$-molecule allows us to use the DFT total energies for $g_{\mathrm{Pt}_{\mathrm{x}} \mathrm{O}_{\mathrm{y}}}^{\text {bulk }}, g_{\mathrm{Pt}}^{\text {bulk }}$, and $E_{\mathrm{O}_{2}}^{\text {tot }}$ to evaluate eq. (11).

Using the experimental value for $\Delta G_{\mathrm{O}_{2}}^{\mathrm{H}_{2} \mathrm{O}}$ and the formation energies of the $\mathrm{Pt}$ bulk-oxides under consideration (see Table 1), which correspond to the first summand on the right side of equation (11), we can formulate the following stability ranges for the different oxides:

$$
\begin{aligned}
& \text { - } \alpha-\mathrm{PtO}_{2}:+1.74 \mathrm{eV}-2 \Delta \phi<\Delta \mu_{\mathrm{H}_{2} \mathrm{O}}<0 \mathrm{eV}, \\
& \text { - } \beta-\mathrm{PtO}_{2}:+1.74 \mathrm{eV}-2 \Delta \phi<\Delta \mu_{\mathrm{H}_{2} \mathrm{O}}<0 \mathrm{eV}, \\
& \text { - } \quad \mathrm{PtO}: \quad+1.97 \mathrm{eV}-2 \Delta \phi<\Delta \mu_{\mathrm{H}_{2} \mathrm{O}}<0 \mathrm{eV} .
\end{aligned}
$$

In the lower plots in Figs. $2-4$ these stability ranges are indicated by shadowing the area where phase separation should occur. Thus, in the following we will focus on the other areas of the phase diagrams.

\section{Results and Discussion}

In the following we will discuss the $(p / T / \phi)$ phase diagrams calculated for the different $\mathrm{Pt}$ bulkoxides, giving insights into the electrode/electrolyte 
interfaces for different environmental conditions. For the relevant systems we will also discuss the corresponding structural characteristics.

In order to evaluate the structure of the electrode surface under high positive electrode potentials, we used the calculated DFT-energies for all different slabs of lowindex Pt bulk-oxide surfaces (see section 2.1) together with equation (7) to obtain the corresponding $(p / T / \phi)$ phase diagrams. The upper 3D-plots in Figures 2-4 show the surface Gibbs energy $\gamma$ as function of the difference in electrode potential $\Delta \phi$ and the chemical potential of the surrounding water $\Delta \mu_{\mathrm{H}_{2} \mathrm{O}}$, resulting in a single plane for every surface structure studied for the particular $\mathrm{Pt}$ bulk-oxide. Since the thermodynamically most stable surface configurations have the lowest surface (interfacial) free energy, the lower plots in Figures 2-4 show the view to the bottom of the corresponding 3Dplot.

Because the chemical potential of the water environment $\Delta \mu_{\mathrm{H}_{2} \mathrm{O}}$ is a function of temperature and pressure/activity, we added the temperature scale, which corresponds to an activity of $a=1$ (representing the a low ion concentration in the), to the plot by using:

$$
\mu_{\mathrm{H}_{2} \mathrm{O}}=\bar{\mu}_{\mathrm{H}_{2} \mathrm{O}}\left(T, a^{0}\right)+k_{\mathrm{B}} T \ln \left(\frac{a_{\mathrm{H}_{2} \mathrm{O}}}{a^{0}}\right),
$$

where $\bar{\mu}_{\mathrm{H}_{2} \mathrm{O}}\left(T, a^{0}\right)$ denotes the standard chemical potential [28] at temperature $T$ and $a_{\mathrm{H}_{2} \mathrm{O}}=1$. In all phase diagrams (Fig. 2-4) room temperature, which is of particular interest for comparison with most experiments, is highlighted with a dashed horizontal line.

As can be seen in all three phase diagrams, the cross point between the dashed horizontal lines and the dashed vertical lines, which represent the $\Delta \phi=0 \mathrm{~V}$ condition, lies within the region of phase separation. This agrees with cyclic-voltammetry measurements $[10,11,12,29,30]$, interpreting the small (or even vanishing) current density at low positive potentials $(\Delta \phi<0.8-0.9 \mathrm{~V})$ as charging of the electric double-layer, consisting of the pure Pt electrode and the surrounding water dipoles. There excess charge is accumulated near the electrode surface, which interacts with the water dipoles and leads to a corresponding orientation of the electrode-near water molecules.

Following the horizontal lines in the phase diagrams to the right (increasing the electrode potentials) shows that above around $1.2 \mathrm{~V}$ for $\alpha-\mathrm{PtO}_{2}$ and $\beta-\mathrm{PtO}_{2}$ and above $1.3 \mathrm{~V}$ for $\mathrm{PtO}$ the bulk-oxide is the thermodynamically stable phase. The transition potentials for $\alpha-\mathrm{PtO}_{2}$ and $\beta-\mathrm{PtO}_{2}$ are also conform with the corresponding $\mathrm{CV}$-measurements, showing the oxideformation to occur above 1.00-1.20 V [10,11,12,29,30], depending on the experimental conditions. separately:

In the following we will discuss each bulk-oxide

$$
\alpha-\mathrm{PtO}_{2}
$$

Among the low-indexed surfaces of this oxide we find that above an electrode potential of $1.2 \mathrm{~V}$ the (001) surface with bulk-composition is most stable [in Fig. 2 denoted as (001)-O], showing only minor relaxation effects. This surface structure corresponds to experimental UHV-measurements of surface-oxides on $\operatorname{Pt}(110)$ support by DFT-calculations [24]. However in case a very thin surface layer is considered, the composition might even change to $\mathrm{PtO}$ instead of $\mathrm{PtO}_{2}$. Further increase of the electrode potential results in a stronger attraction of water molecules towards the surface. Due to this interaction at electrode potentials above $2.0 \mathrm{~V}$, the surface structure would theoretically change to a $\alpha-\mathrm{PtO}_{2}(011)$ surface with an enrichment of oxygen, respectively $\mathrm{O}^{2-}$ on the surface. Since all $\mathrm{Pt}$ atoms are already saturated with $\mathrm{O}$ atoms, the excess oxygens form $\mathrm{O}=\mathrm{O}$ dimers on the surface with $d(\mathrm{O}=\mathrm{O})=1.31 \AA$, what is comparable to the double bond of an $\mathrm{O}_{2}$ adsorbed on pure $\operatorname{Pt}(111)$ (1.27 $\AA$ ) [31].

$$
\beta-\mathrm{PtO}_{2}
$$

Comparable to $\alpha-\mathrm{PtO}_{2}$, at room temperature and an electrode potential around $1.2 \mathrm{~V}$, which exactly corresponds to the transition of the phase separated system and the bulk-oxide, the (110) surface again with bulk-composition is most stable. In the lower plot of Fig. 3 this corresponds to the phase directly below the black line marking this phase transition. Regarding the structure of this surface the $\mathrm{Pt}-\mathrm{O}$ distances at the surface show only minor deviations from the corresponding value in the bulk $d(\mathrm{Pt}-\mathrm{O})=2.05 \AA$. Similarly the $\mathrm{O}-\mathrm{O}$ distances shorten by less than $3 \%$ compared to their bulk analogs. Slight increase of the electrode potential accumulates further oxygen at the surface. The structure being most stable up to $\sim 1.4 \mathrm{~V}$ is enriched by one additional oxygen atom per surface unit cell. Induced by these excess atoms (see $\mathrm{O} 3$ in Figure 3), which are not compensated by corresponding platinums, structural changes on the surface can be observed. Besides forming a covalent bond to a Pt atom near the surface $(d=2.09 \AA)$ a connection to an adjacent oxygen atom is formed. The O2-O3 bond length of $1.41 \AA$ is $0.19 \AA$ larger than the atom separation in a gas-phase $\mathrm{O}_{2}$ molecule, but comparable to the distance in the $\mathrm{OOH}$ radical and $\mathrm{H}_{2} \mathrm{O}_{2}$ molecule, respectively [31]. Therefore, a single covalent bond establishes between $\mathrm{O} 2$ and $\mathrm{O} 3$. Here it should be mentioned, that in our calculations the excess surface charge, which is induced by the increasing electrode potential, is not treated explicitly. However, the expected repulsion between oxygens at or near the surface might prevent covalent $\mathrm{O}-\mathrm{O}$ bonds to form on the surface. The influence of the excess surface charge will be subject of further investigations.

Above $1.4 \mathrm{~V}$ the attraction of the water dipoles increases, finally stabilizing a second excess oxygen per surface unit cell. Caused by this additional atom the bond length between $\mathrm{O} 3$ and $\mathrm{O} 4$ reduces to $1.27 \AA$, which is exactly the value obtained for the $\mathrm{O}=\mathrm{O}$ double bond of an $\mathrm{O}_{2}$-dimer adsorbed on pure $\mathrm{Pt}(111)$. 


\section{PtO}

Since the PtO bulk-oxide is less stable than $\alpha$ $\mathrm{PtO}_{2}$ or $\beta-\mathrm{PtO}_{2}$, reaching the stability region of the bulkoxide requires slightly higher electrode potentials $(\Delta \phi>$ $1.3 \mathrm{~V})$. From this value up to $2.5 \mathrm{~V}$ we find the $\mathrm{PtO}(100)$ structure to be thermodynamically most stable (see Fig. 4). Interestingly the first surface layer shows a $\mathrm{PtO}_{2}$ composition, which is similar to what was obtained for the previous bulk-oxides. However, including the underlying layer of $\mathrm{Pt}$ atoms results in a $\mathrm{PtO}$ composition as in the bulk. This surface structure again agrees with a low-coverage surface-oxide found on $\mathrm{Pt}(110)$ by STMmeasurements under UHV conditions [24]. Compared to the layer separation within the bulk, the surface layer shows a small relaxation of $\sim 4 \%$ towards the surface.

Equivalent to the other bulk-oxides already discussed, further increase of the electrode potential would theoretically lead to on O-enrichment at the surface, stabilizing the $\mathrm{PtO}(010)-\mathrm{O}_{2}$ surface, which is terminated by two $\mathrm{O}$-atoms. Since in this system no $\mathrm{O}_{2}$ formation can be observed, strong bonds are established between the surface $\mathrm{O}$-atoms and the Pt-atoms beneath, resulting in a reduction of the $\mathrm{Pt}-\mathrm{O}$ distance by $0.3 \AA$ compared to the bulk. Consequently, the layer of $\mathrm{Pt}$ atoms near the surface separates from the underlying bulk also by $0.3 \AA$.

On the basis of the previous discussion, we suggest that at not too high electrode potentials the oxide growth proceeds by first forming a thin $\alpha-\mathrm{PtO}_{2}(001)$ or $\mathrm{PtO}(100)$ surface oxide with a PtO composition (at very high electrode potentials a $\mathrm{PtO}_{2}$-composition is expected). Further growing then results in a $\alpha-\mathrm{PtO}_{2}(001)$ or $\beta-\mathrm{PtO}_{2}(110)$ bulk-oxide. Therefore, our results agree with experimental observations along the oxidation mode $[13,14,15]$, but in addition specify the specific surface structure. Along the reduction mode Tremiliosi-Filho et al. [15] found the opposite growth mechanism to occur. Although this seems to be in contrast with our results, one has to consider that the surface structures discussed in the present work correspond to thermodynamically stable phases. Thus, any energetic barriers the system has to overcome in order to reach thermodynamic equilibrium are not included. Those studies would require extensive kinetic simulations for which the results presented here might be used as starting point.

\section{Summary and Outlook}

A combination of density functional theory calculations and the extended $a b$ initio atomistic thermodynamics approach was used to study the structure of Pt-electrodes at elevated electrode potentials. First we described the methodology by initially starting with the gas-phase system and then transferring this to electrochemical conditions in which the Pt-electrode is in contact with an aqueous solution containing electrolyte ions, which should fulfill the condition of no specific ion adsorption. Since our interest is in the potential range where the oxide-formation occurs, we afterwards formulated the environmental conditions under which the corresponding $\mathrm{Pt}$ bulk-oxides represent the thermodynamically stable phases. Here we considered the stable bulk-oxides reported in literature and found that under experimental temperature conditions ( $T=298 \mathrm{~K})$ and an activity of $a=1$, electrode potentials above $1.2 \mathrm{~V}$ are required to stabilize $\alpha-\mathrm{PtO}_{2}$ and $\beta-\mathrm{PtO}_{2}$, while $1.3 \mathrm{~V}$ is the transition for bulk-PtO, which is in good agreement with experimental CV-measurements.

To obtain a deeper insight into the structure of the electrode surface at these potentials, we afterwards performed DFT-calculations on all low-index surfaces of the three Pt bulk-oxides, distinguishing between various possible surface terminations. Then these results were used to evaluate the corresponding $(p, T, \phi)$-phase diagrams. For all three systems it was found that at electrode potentials around (but above) the transition potentials mentioned above, the surface prefers a $\mathrm{PtO}_{2}$ composition within the first layer. However, considering thin oxide layers (1-2 layers) the $\alpha-\mathrm{PtO}_{2}$ - as well as the $\mathrm{PtO}-$ system indicate the possibility of a stable phase having a $\mathrm{PtO}$ composition and a $\mathrm{PtO}(100)$ structure. However, with increasing thickness of the oxide layer the higher bulk stabilities of $\alpha-\mathrm{PtO}_{2}$ and $\beta-\mathrm{PtO}_{2}$ compared to $\mathrm{PtO}$ will result in the formation of $\alpha-\mathrm{PtO}_{2}$ and $/$ or $\beta-\mathrm{PtO}_{2}$ oxides having $\alpha-\mathrm{PtO}_{2}(001)$ and/or $\beta-\mathrm{PtO}_{2}(110)$ surface structures.

As expected, for all three oxides applying higher positive electrode potentials led to an enrichment of oxygen at the surface. While in case of $\beta-\mathrm{PtO}_{2}$ the (110) surface is already densely packed, this surface structure remains the thermodynamically stable one even at elevated potentials. In contrast $\alpha-\mathrm{PtO}_{2}$ and $\mathrm{PtO}$, which start with their (001), respectively (100) surfaces stabilize more densely packed surfaces at higher electrode potentials: $\alpha-\mathrm{PtO}_{2}(011)$ and $\mathrm{PtO}(010)$. In case of $\alpha-\mathrm{PtO}_{2}$ and $\beta-\mathrm{PtO}_{2}$ the high oxygen enrichment at the surfaces partially led to the formation of $\mathrm{O}_{2}$.

While in the present work we focused on the interfacial structure of Pt-electrodes in the oxideformation region, at electrode potentials around the value where oxide-formation starts, it is also reasonable to consider the formation of thin oxide layers on pure $\mathrm{Pt}$ electrodes (not bulk-oxides). Besides the possibility of forming thermodynamically stable thin oxide layers, those structures are also relevant for the process of oxideformation itself. In this context, forthcoming investigations will focus on using the surface structures evaluated within the present work to find stable thin oxide layers.

\section{Acknowledgements}

Support by the "Fonds der Chemischen Industrie" (VCI) is greatly acknowledged. 


\section{Figures}

Figure 1: Bulk-unitcells of the three most stable Pt-oxides: $\alpha-\mathrm{PtO}_{2}, \beta-\mathrm{PtO}_{2}, \mathrm{PtO}$. Dark spheres indicate Pt atoms, while light ones are Oxygens.
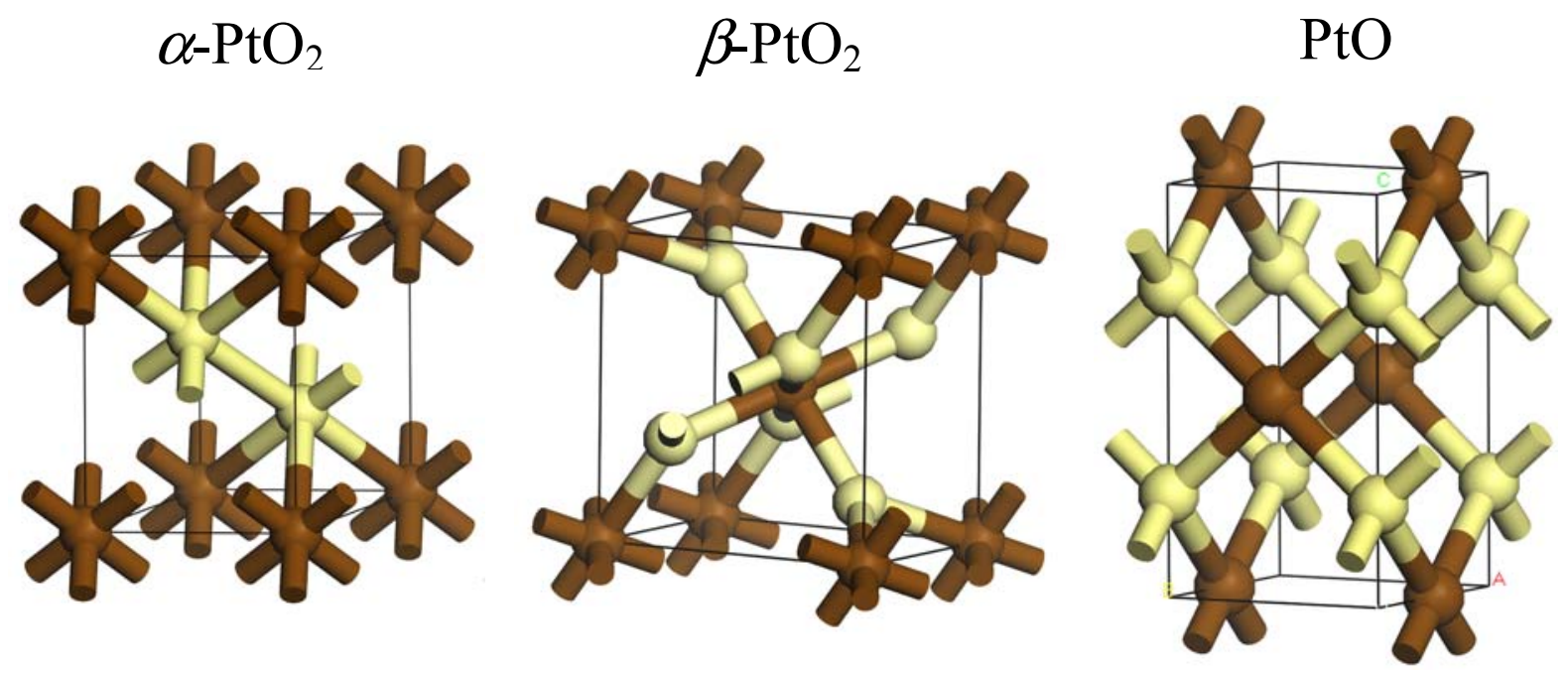
Figure 2: $(p / T / \phi)$-phase diagram for all low-index surfaces of $\alpha-\mathrm{PtO}_{2}$. The upper plot shows the surface Gibbs energy $\gamma$ as function of $\Delta \mu_{\mathrm{H} 2 \mathrm{O}}$ and $\Delta \phi$, while the lower plot shows the bottom view, indicating the thermodynamically most stable structures (Pt: red; O: yellow). In addition the temperature scale, which corresponds to $a=1$ ( $p=1 \mathrm{~atm}$.), is given on the right side of the phase diagram. The surface models are named with their orientations and surface terminations.
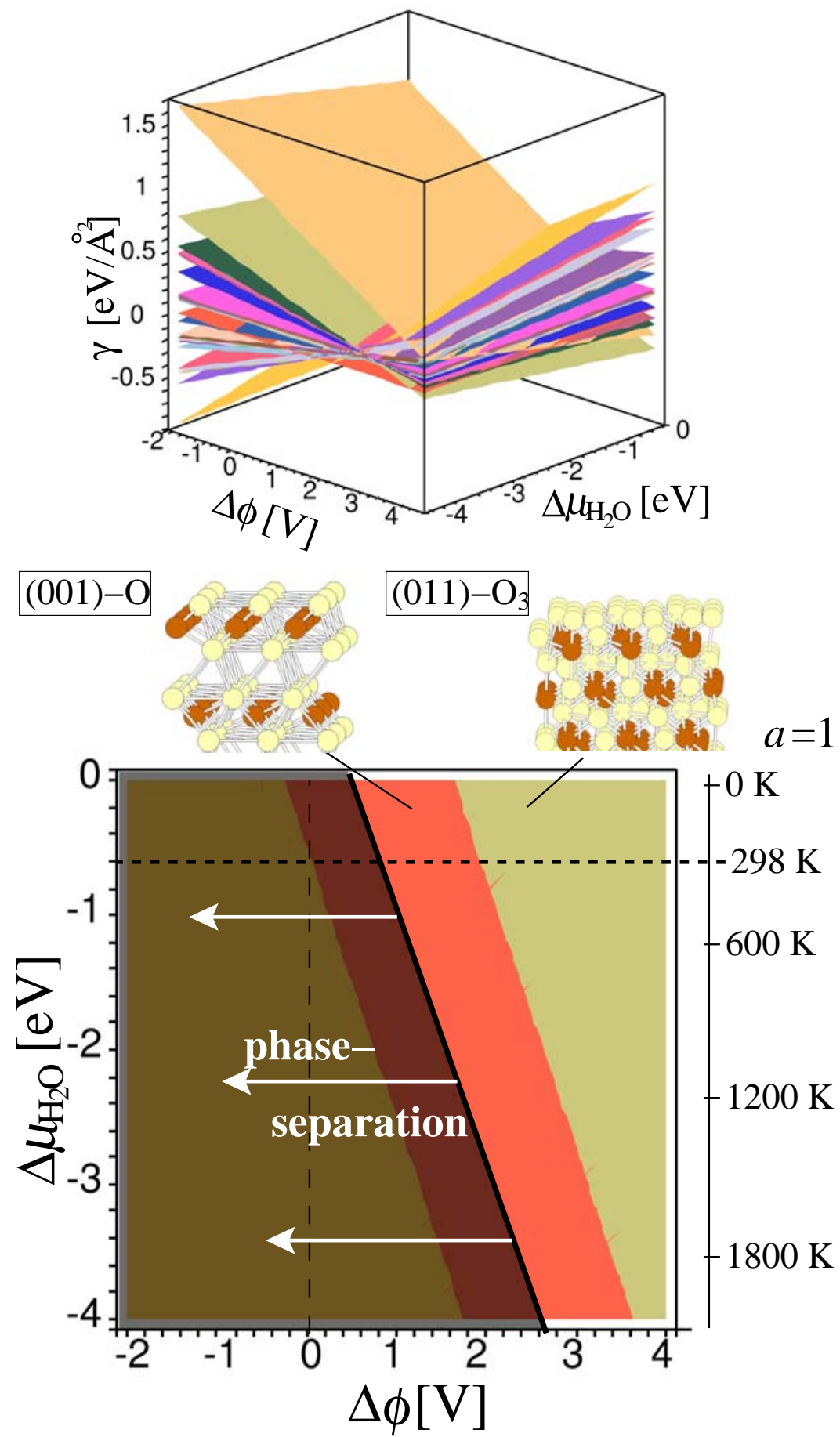
Figure 3: $(p / T / \phi)$-phase diagram for all low-index surfaces of $\beta-\mathrm{PtO}_{2}$. The upper plot shows the surface Gibbs energy $\gamma$ as function of $\Delta \mu_{\mathrm{H} 2 \mathrm{O}}$ and $\Delta \phi$, while the lower plot shows the bottom view, indicating the thermodynamically most stable structures (Pt: red; O: yellow). In addition the temperature scale, which corresponds to $a=1$ ( $p=1 \mathrm{~atm}$.), is given on the right side of the phase diagram. The surface models are named with their orientations and surface terminations.
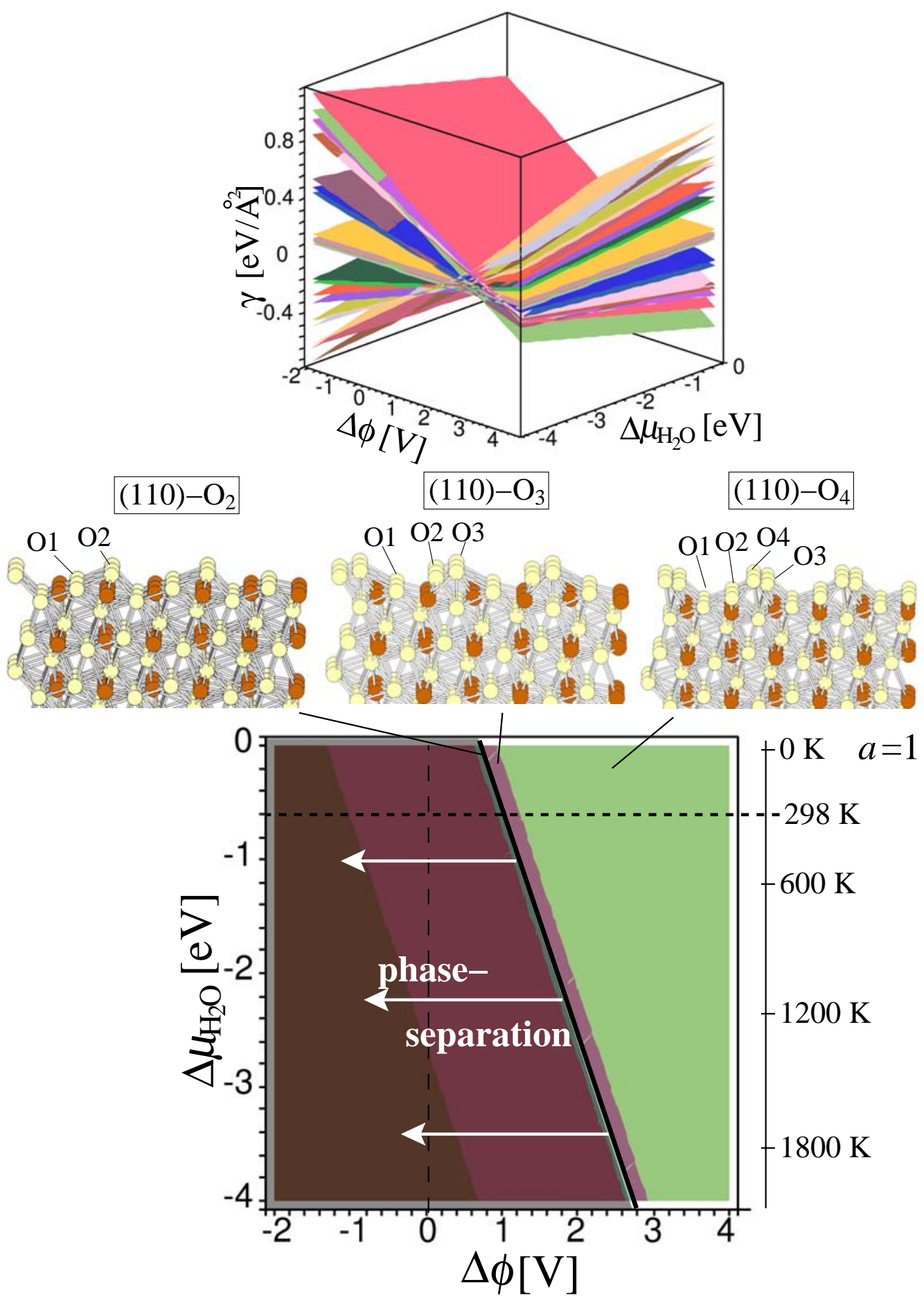
Figure 4: $(p / T / \phi)$-phase diagram for all low-index surfaces of PtO. The upper plot shows the surface Gibbs energy $\gamma$ as function of $\Delta \mu_{\mathrm{H} 2 \mathrm{O}}$ and $\Delta \phi$, while the lower plot shows the bottom view, indicating the thermodynamically most stable structures (Pt: red; O: yellow). In addition the temperature scale, which corresponds to $a=1$ ( $p=1 \mathrm{~atm}$.), is given on the right side of the phase diagram. The surface models are named with their orientations and surface terminations.

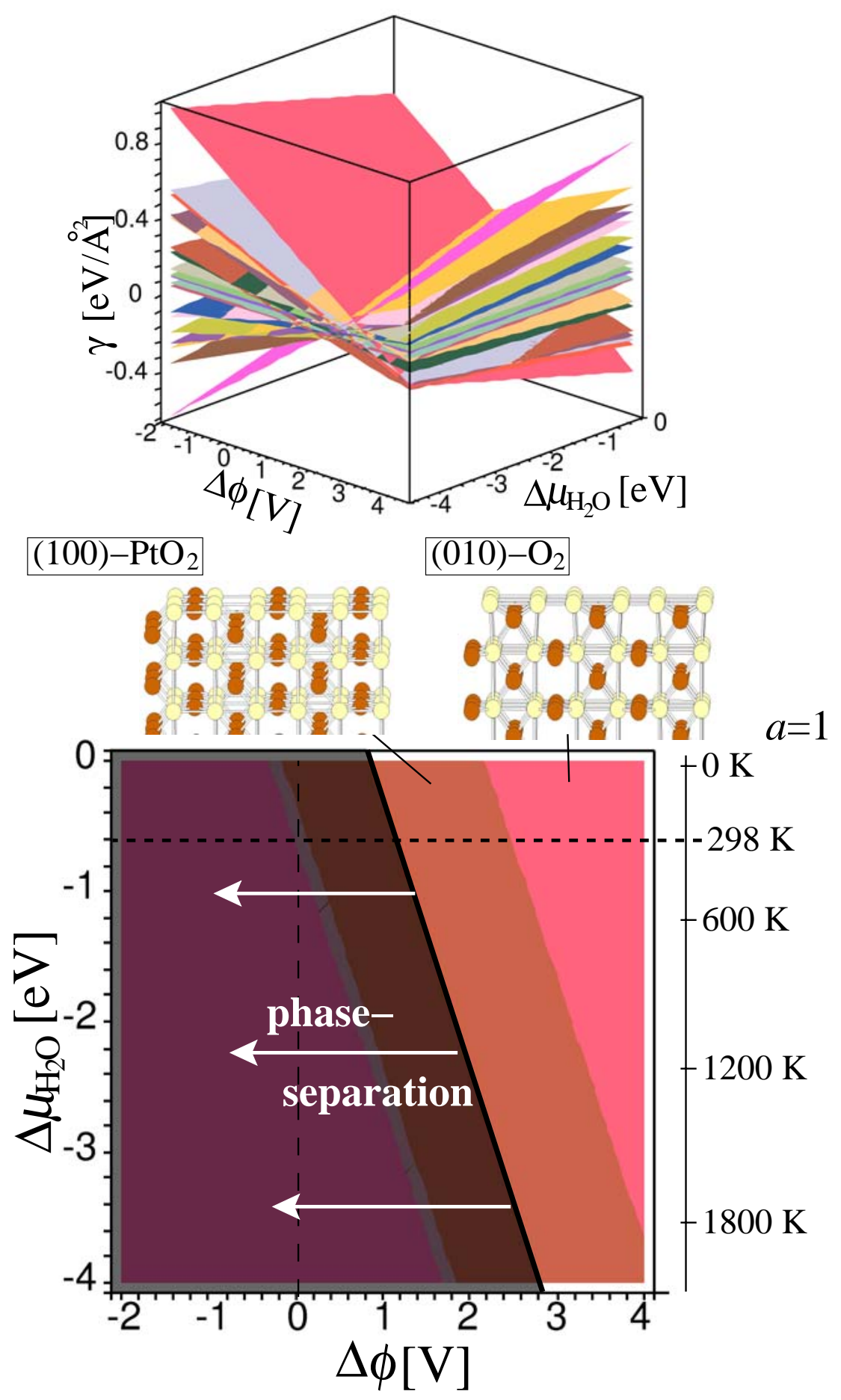




\section{Tables}

Table 1: Crystal structures and formation energies (per O-atom) of different bulk-oxides. The energy values are referenced to Pt-bulk and molecular $\mathrm{O}_{2}$.

\begin{tabular}{|c|c|c|c|c|c|c|c|}
\hline \multirow{3}{*}{$\begin{array}{l}\text { System } \\
\alpha-\mathrm{PtO}_{2}\end{array}$} & \multirow{3}{*}{$\begin{array}{c}\text { Space } \\
\text { Group }\end{array}$} & \multicolumn{4}{|c|}{ Crystal Structure } & \multicolumn{2}{|c|}{$\Delta \mathbf{H}_{\mathrm{f}}[\mathrm{eV}]$ (per O-atom) } \\
\hline & & \multicolumn{2}{|c|}{ calculated } & \multicolumn{2}{|c|}{ experiment [32] } & calculated & experiment [23] \\
\hline & & $\begin{array}{l}a=3.153 \AA \\
b=3.153 \AA \\
c=4.545 \AA\end{array}$ & $\begin{array}{r}\alpha=90^{\circ} \\
\beta=90^{\circ} \\
\gamma=120^{\circ}\end{array}$ & $\begin{array}{l}a=3.100 \AA \\
b=3.100 \AA \\
c=4.161 \AA\end{array}$ & $\begin{array}{r}\alpha=90^{\circ} \\
\beta=90^{\circ} \\
\gamma=120^{\circ}\end{array}$ & -0.72 & -0.69 \\
\hline$\beta-\mathrm{PtO}_{2}$ & Pnnm & $\begin{array}{l}a=4.631 \AA \\
b=4.523 \AA \\
c=3.193 \AA\end{array}$ & $\begin{array}{l}\alpha=90^{\circ} \\
\beta=90^{\circ} \\
\gamma=90^{\circ}\end{array}$ & $\begin{array}{l}a=4.484 \AA \\
b=4.539 \AA \\
c=3.136 \AA\end{array}$ & $\begin{array}{l}\alpha=90^{\circ} \\
\beta=90^{\circ} \\
\gamma=90^{\circ}\end{array}$ & -0.72 & -0.69 \\
\hline PtO & P42mmc & $\begin{array}{l}a=3.133 \AA \\
b=3.133 \AA \\
c=5.450 \AA\end{array}$ & $\begin{array}{l}\alpha=90^{\circ} \\
\beta=90^{\circ} \\
\gamma=90^{\circ}\end{array}$ & $\begin{array}{l}a=3.043 \AA \\
b=3.043 \AA \\
c=5.345 \AA\end{array}$ & $\begin{array}{l}\alpha=90^{\circ} \\
\beta=90^{\circ} \\
\gamma=90^{\circ}\end{array}$ & -0.49 & - \\
\hline $\mathbf{P t}_{3} \mathbf{O}_{4}$ & Pm-3n & $\begin{array}{l}a=5.678 \AA \\
b=5.678 \AA \\
c=5.678 \AA\end{array}$ & $\begin{array}{l}\alpha=90^{\circ} \\
\beta=90^{\circ} \\
\gamma=90^{\circ}\end{array}$ & $\begin{array}{l}a=5.585 \AA \\
b=5.585 \AA \\
c=5.585 \AA\end{array}$ & $\begin{array}{l}\alpha=90^{\circ} \\
\beta=90^{\circ} \\
\gamma=90^{\circ}\end{array}$ & -0.68 & - \\
\hline
\end{tabular}




\section{References}

[1] B. J. Cruickshank, D. D. Sneddon, A. A. Gewirth, Surf. Sci. Lett. 281 (1993) 308.

[2] D. M. Kolb, Angew. Chem. Int. Ed. 40 (2001) 1162.

[3] M. Wilms, P. Broeckmann, C. Stuhlmann, K. Wandelt, Surf. Sci. 416 (1998) 121.

[4] D. M. Kolb, Surf. Sci. 500 (2002) 702.

[5] T. Jacob, Electrochim. Acta, 52 (2006) 2229.

[6] D. M. Kolb, L. A. Kibler, Z. Phys. Chem. 217 (2003) 1265.

[7] T. Iwasita, F. C. Nart, Prog. Surf. Sci. 55 (1997) 271.

[8] H. Angerstein-Kozlowska, B. E. Conway, W. B. A. Sharp, J. Electroanal. Chem. 43 (1973) 9.

[9] B. E. Conway, Prog. Surf. Sci. 49 (1995) 331.

[10] J. Clavilier, J. M. Orts, R. Gomez, J. M. Feliu, A. Aldaz, J. Electroanal. Chem. 404 (1996) 281.

[11] G. Jerkiewicz, G. Vatankhah, J. Lessard, M. P. Soriaga, Y.-S. Park, Electrochim. Acta 49 (2004) 1451.

[12] G. Stalnionis, L. Tamašauskaitė-Tamašiūnaitè, V. Pautienienè, Z. Jusys, J. Electroanal. Chem. 590 (2006) 198.

[13] D. Gilroy, B. E. Conway, Can. J. Chem. 46 (1968) 875.

[14] G. C. Allen, P. M. Tacker, A. Capon, R. Parsons, J. Electroanal. Chem. 50 (1974) 335.

[15] G. Tremiliosi-Filho, G. Jerkiewicz, B. E. Conway, Langmuir 8 (1992) 658.

[16] C. D Taylor, S. A. Wasileski, J.-S. Filhol, M. Neurock, Phys. Rev. B 73 (2006) 165402.

[17] T. Jacob, M. Scheffler, Phys. Rev. Lett., submitted (2007).

[18] P. A. Schultz, (unpublished); A description of the method is in: P. J. Feibelman, Phys. Rev. B, 35 (1987) 2626.

[19] C. Verdozzi, P.A. Schultz, R. Wu, A.H. Edwards, N. Kioussis, Phys. Rev. B 66 (2002) 125408.

[20] J. P. Perdew, K. Burke, M. Ernzerhof, Phys. Rev. Lett. 88 (1996) 3865.

[21] D. R. Hamann, Phys. Rev. B 40 (1989) 2980.

[22] S. G. Louie, S. Froyen, M.L. Cohen, Phys. Rev. B 26 (1982) 1738.

[23] The Oxide Handbook, edited by G. V. Samsonov, IFI/Plenum, New York (1973).

[24] W. X. Li, L. Österlund, E. K. Vestergaard, R. T. Vang, J. Matthiesen, T. M. Pedersen, E. Lægsgaard, B. Hammer, F. Besenbacher, Phys. Rev. Lett. 93 (2004) 146104.

[25] K. Reuter, M. Scheffler, Phys. Rev. B 68 (2003) 045407.

[26] V. Climent, R. Gómez, José M. Orts, J. M. Feliu, J. Phys. Chem. B 110 (2006) 11344.

[27] P. W. Atkins, Physikalische Chemie, Wiley-VCH, Weinheim (1990).

[28] D. R. Stull, H. Prophet, JANAF Thermochemical Tables, $2^{\text {nd }}$ edition, U.S. National Bureau of Standards (U.S. EPO, Washinghton D.C., 1971).

[29] K. Sashikata, N. Furuya, K. Itaya, J. Vac. Sci. Technol. B 9 (1991) 457.

[30] D. Armand, J. Clavilier, J. Electroanal. Chem. 270 (1989) 331.

[31] T. Jacob, Fuel Cells, 6 (2006) 159.

[32] H. R. Hoekstra, S. Siegel, F. X. Gallagher, Adv. Chem. Series 98 (1971) 39; K.-J. Range, F. Rau, U. Klement, A. M. Heyns, Mat. Res. Bulletin 22 (1987) 1541; W. J. Moore, L. Pauling, J. Am. Chem. Soc. 63 (1941) 1392; O. Muller, R. Roy J. Less-Common Metals 16 (1968) 129. 